EPJ Web of Conferences 31, 00004 (2012)

DOI: $10.1051 /$ epjconf/20123100004

C) Owned by the authors, published by EDP Sciences - SIF, 2012

\title{
Constrained caloric curves for hot nuclei
}

\author{
B. Borderie ${ }^{1}$, S. Piantelli ${ }^{2}$, M.-F. Rivet ${ }^{1}$ and Ad. R. Raduta ${ }^{3}$ \\ for the INDRA COLLABORATION
}

\author{
${ }^{1}$ Institut de Physique Nucléaire, CNRS/IN2P3, Univ. Paris-Sud 11, \\ Orsay, France \\ ${ }^{2}$ Sezione INFN, Sesto Fiorentino (Fi), Italy \\ ${ }^{3}$ National Institute for Physics and Nuclear Engineering, \\ Bucharest-Măgurele, Romania
}

\begin{abstract}
Simulations based on experimental data obtained from multifragmenting quasi-fused nuclei produced in central ${ }^{129} \mathrm{Xe}+{ }^{n a t} \mathrm{Sn}$ collisions have been used to deduce event by event freeze-out properties on the thermal excitation energy range 4-12 AMeV. From such properties, information on constrained caloric curves has been derived. At constant average volumes caloric curves exhibit a monotonous behaviour whereas for constrained pressures backbending is observed. Such results support the existence of a first order phase transition for hot nuclei.
\end{abstract}

\section{Introduction}

One of the most important challenges of heavy-ion collisions at intermediate energies is the identification and characterization of the nuclear liquid-gas phase transition for hot nuclei, which was earlier theoretically predicted for nuclear matter [1]. In the last fifteen years a big effort to accumulate experimental indications of the phase transition has been made. Statistical mechanics of finite systems appeared as a key issue to progress and propose new first-order phase transition signatures related to thermodynamic anomalies 
like negative microcanonical heat capacity and bimodalities [2-4]. Correlated temperature and excitation energy measurements, commonly termed caloric curves, were the first studied possible signatures of phase transition. However in spite of the observation of a plateau in some caloric curves, no decisive conclusion was extracted $[1,5-7]$. The reason is that experimentally it is not possible to explore the caloric curves at constant pressure or constant average volume, which is required for an unambiguous phase transition signature. Indeed, theoretical studies show that if many different caloric curves can be generated depending on the path followed in the thermodynamical variable plane, constrained caloric curves exhibit different behaviours in presence of a first order phase transition: a monotonous evolution at constant average volume and a back bending of curves at constained pressures $[8,9]$. With the help of a simulation able to carefully reproduce all the experimental observables measured for hot nuclei formed in central collisions (quasi-fused systems, QF, from ${ }^{129} \mathrm{Xe}+{ }^{n a t} \mathrm{Sn}, 32-50 \mathrm{AMeV}$ ), event by event properties at freeze-out were restored and used to build constrained caloric curves. The definition of pressure in the microcanonical framework is presented in section 2 . Then, in section 3 , simulations to recover freeze-out properties of multifragmentation events are briefly described [10,11]. The deduced constrained caloric curves are presented in section 4 . In section 5 , the classical thermometer derived from simulations is discussed and a more quantum approach is proposed to produce final constrained caloric curves.

\section{Pressure in the microcanonical framework}

Let us consider a gas of weakly interacting fragments (i.e. they interact only by Coulomb and excluded volume), which corresponds to the freezeout configuration. Within a microcanonical ensemble, the statistical width of a configuration $C$, defined by the mass, charge and internal excitation energy of each of the constituting $M_{C}$ fragments, writes

$$
\begin{array}{r}
W_{C}(A, Z, E, V)=\frac{1}{M_{C} !} \chi V^{M_{C}} \prod_{n=1}^{M_{C}}\left(\frac{\rho_{n}\left(\epsilon_{n}\right)}{h^{3}}\left(m A_{n}\right)^{3 / 2}\right) \\
\frac{2 \pi}{\Gamma\left(3 / 2\left(M_{C}-2\right)\right)} \frac{1}{\sqrt{(\operatorname{det} I)}} \frac{(2 \pi K)^{3 / 2 M_{C}-4}}{(m A)^{3 / 2}},
\end{array}
$$

where $I$ is the moment of inertia, $K$ is the thermal kinetic energy, $V$ is the freeze-out volume and $\chi V^{M_{C}}$ stands for the free volume or, equivalently, accounts for inter-fragment interaction in the hard-core idealization. 
The microcanonical equations of state are

$$
\begin{gathered}
T=\left.\left(\frac{\partial S}{\partial E}\right)^{-1}\right|_{V, A}, \\
P / T=\left.\left(\frac{\partial S}{\partial V}\right)\right|_{E, A}, \\
-\mu / T=\left.\left(\frac{\partial S}{\partial A}\right)\right|_{E, V} .
\end{gathered}
$$

Taking now into account that $S=\ln Z=\ln \sum_{C} W_{C}$ and that $\partial W_{C} / \partial V=\left(M_{C} / V\right) W_{C}$ it comes out that

$$
\begin{aligned}
P / T=\left(\frac{\partial S}{\partial V}\right) & =\frac{1}{\sum_{C} W_{C}} \sum_{C} \frac{\partial W_{C}}{\partial V} \\
& =\frac{1}{V} \frac{\sum_{C} M_{C} W_{C}}{\sum_{C} W_{C}}=\frac{\left\langle M_{C}\right\rangle}{V} .
\end{aligned}
$$

The microcanonical temperature is also easily deduced from its statistical definition [12]:

$$
\begin{aligned}
T=\left(\frac{\partial S}{\partial E}\right)^{-1} & =\left(\frac{1}{\sum_{C} W_{C}} \sum_{C} W_{C}\left(3 / 2 M_{C}-5 / 2\right) / K\right)^{-1} \\
& =<\left(3 / 2 M_{C}-5 / 2\right) / K>^{-1} .
\end{aligned}
$$

where $K$ is the total thermal kinetic energy of the system at freeze-out.

As $M_{C}$, the total multiplicity at freeze-out, is large, the pressure $P$ can be well approximated by

$$
P=\frac{2}{3} \frac{<K>}{V} .
$$

\section{Event by event freeze-out properties}

Starting from all the available asymptotic experimental information (charged particle energy spectra, average and standard deviation of fragment velocity spectra and calorimetry) of selected QF sources produced in central ${ }^{129} \mathrm{Xe}+{ }^{\text {nat }} \mathrm{Sn}$ collisions which undergo multifragmentation, a simulation was performed to reconstruct freeze-out properties event by event $[10,11]$. The method requires data with a very high degree of completeness (total detected charge $\geq 93 \%$ of the total charge of the system), which is crucial for a good estimate of Coulomb energy. QF sources are reconstructed, event by 
event, in the reaction centre of mass, from all the fragments and twice the charged particles emitted in the range $60-120^{\circ}$ in order to exclude a major part of pre-equilibrium emission. Dressed excited fragments, which in flight statistically deexcite, and particles at freeze-out are described by spheres at normal density. Four free parameters are used to recover the data at each incident energy: the percentage of measured particles which were evaporated from primary fragments, the collective radial energy, a minimum distance between the surface of products at freeze-out and a limiting temperature for fragments. Such a limiting temperature, related to the vanishing of level density for fragments [13], was mandatory to reproduce the observed widths of fragment velocities. Indeed, Coulomb repulsions plus collective energy plus thermal kinetic energy (directed at random) plus spreading due to fragment decays are responsible for about $60-70 \%$ of the observed widths. By introducing a limiting temperature for fragments, thermal kinetic increases, due to energy conservation, which produces the missing percentage for the widths of final velocity distributions. The agreement between experimental and simulated velocity/energy spectra for fragments/charged particles and for the different beam energies is quite remarkable (see figures 3 and 5 of [11]). Relative velocities between fragment pairs were also compared through reduced relative velocity correlation functions $[14,15]$ (see figure 4 of [11] ). Again a good agreement is obtained between experimental data and simulations, which indicates that the retained method (freeze-out topology built up at random) and parameters are sufficiently relevant to correctly describe the freeze-out configurations, including volumes. From the simulations it is then possible to recover, event by event, the different quantities needed to build constrained caloric curves, namely the thermal excitation energy of QF hot nuclei, $E^{*}$ (total excitation minus collective energy), the kinetic temperature $T_{k i n}$ at freeze-out, the freeze-out volume $V$ (see envelopes of figure 8 from [11]) and the total thermal kinetic energy at freeze-out $K$.

\section{Constrained caloric curves}

Constrained caloric curves, which correspond to correlated values of $E^{*}$ and $T_{\text {kin }}$ have been determined for QF hot nuclei with $\mathrm{Z}$ restricted to the range 80-100, which corresponds to the A domain 194-238 in order to reduce any possible effect of mass variation on caloric curves [7]. In fig. 1 (left) we have constructed caloric curves for different average freeze-out volumes corresponding to the three ranges 3.0-4.0 $V_{0}, 4.0-5.0 V_{0}$ and 5.0-6.0 $V_{0}$ where $V_{0}$ correspond to the volume of the QF nuclei at normal density. As theoreti- 

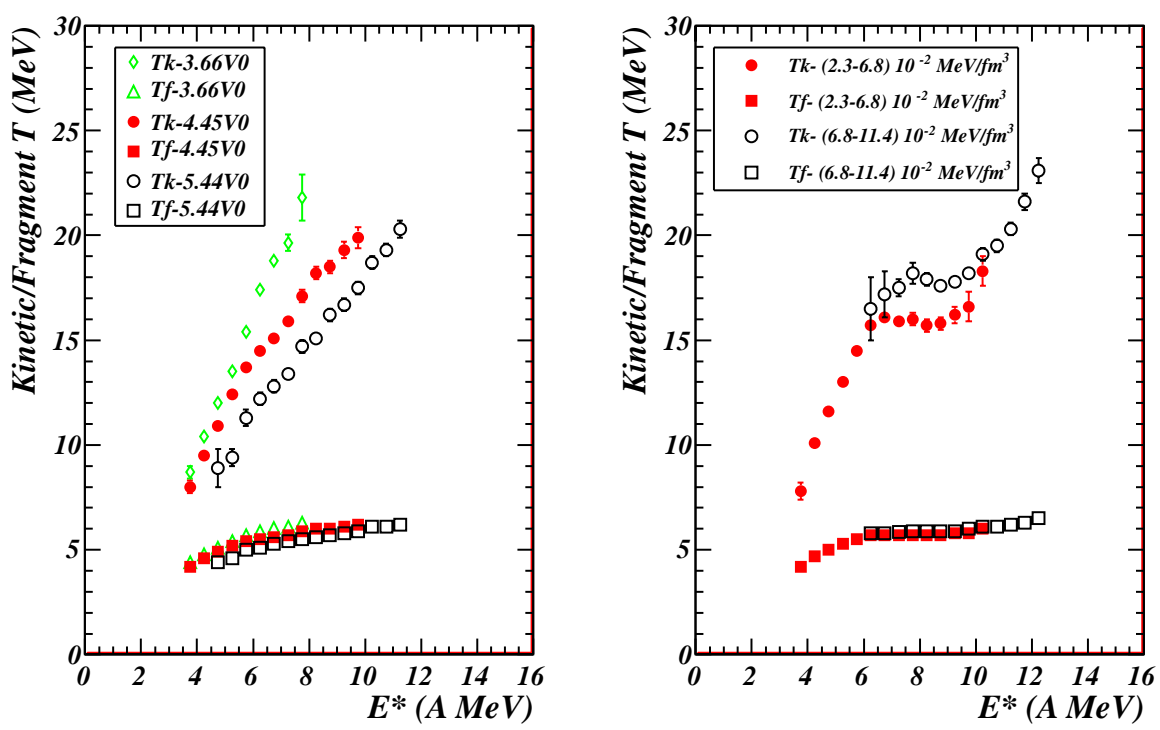

Figure 1: (Colour online) Caloric curves (kinetic temperature versus thermal excitation energy) constrained at average volumes (left) and for selected ranges of pressure (right) and the corresponding internal temperatures for fragments.

cally expected a monotonous behaviour of caloric curves is observed. Curves for fragment temperatures are also shown in the figure. Fig. 1 (right) shows the caloric curves when pressure has been constrained within two domains: 2.3-6.8 and 6.8-11.4 $10^{-2} \mathrm{MeVfm}^{-3}$. Backbending are clearly seen and moreover the qualitative evolution of curves with increasing pressure exactly corresponds to what is theoretically predicted with a microcanonical lattice gas model [8]. As in fig. 1 (left) the evolution of fragment temperatures is also reported. By extrapolating at higher pressures caloric curves of fig. 1 (right), one could infer a critical temperature around $20 \mathrm{MeV}$. Such a value is within the range calculated for infinite nuclear matter whereas a much lower value is expected for nuclei in relation with surface and Coulomb effects [1].

\section{Discussion and conclusion}

In simulations, Maxwell-Boltzmann distributions are used to reproduce the kinetic properties of data and consequently the deduced temperatures, $T_{k i n}$, are classical. At that point, one may wonder if $T_{k i n}$ is a relevant thermometer. First of all one can calculate temperatures with the well known "He/Li thermometer" used in [5] keeping the proposed prefactor 16. The low multi- 


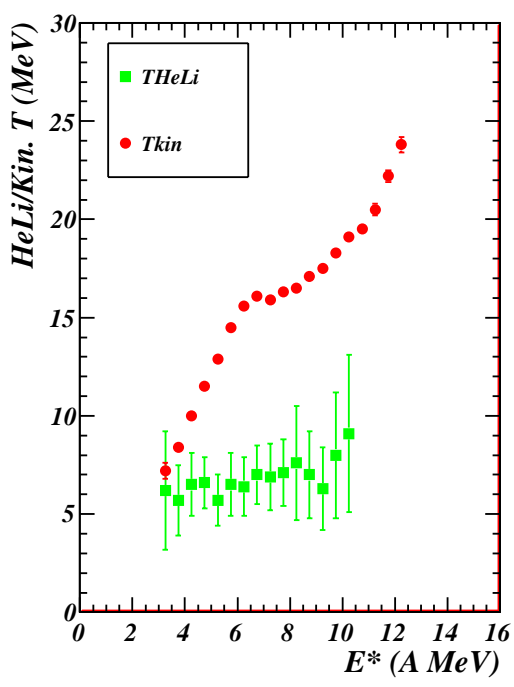

Figure 2: (Colour online) Caloric curves with two different thermometers: $T_{k i n}$ and $T_{H e L i}$ (see text).

plicities experimentally observed for ${ }^{3} \mathrm{He}$ and Li isotopes and the high degree of completeness required for QF nuclei, which reduces the number of events, introduce large temperature error bars and prevent any construction of constrained caloric curves. However one can build the caloric curve without constraints to be compared to the same curve using $T_{k i n}$. Results are displayed in fig. 2. $T_{\mathrm{HeLi}}$ exhibits a plateau around $6-7 \mathrm{MeV}$ up to $E^{*} \approx 9-10$ $\mathrm{AMeV}$ in agreement with [7] whereas $T_{\text {kin }}$ increases from 7 to $17 \mathrm{MeV}$ in the same $E^{*}$ range. Secondly, and most important, we must recall that dispersions in fragment transverse momentum spectra generated in projectile fragmentation were succesfully explained by adding the Fermi momenta of individual nucleons in fragments [16], which is a consequence of the quantum nature of nucleons. For multifragmentation reactions a similar approach was first proposed in [17] to qualitatively explain the different temperatures obtained from various observables (kinetic energies, level populations, isotope ratios). Very recently a thermometer based on quantum fluctuations was proposed [18]. Using Fermi-Dirac distribution, proton momentum fluctuations at finite temperature are calculated and show an evident difference with respect to a classical calculation. Even at zero temperature and ground state density, fluctuations arise from the Fermi motion whereas at sufficient $E^{*}$ protons at freeze-out are emitted with quantum fluctuations. The last step of this work will be to produce constrained caloric curves using this thermometer for momentum fluctuations of protons emitted at freeze-out, and related densities to confirm, we hope, the present results. 


\section{References}

[1] B. Borderie, J. Phys. G: Nucl. Part. Phys. 28 (2002) R217.

[2] P. Chomaz, F. Gulminelli et al. (eds.) vol. 30 of Eur. Phys. J. A, Springer, 2006.

[3] B. Borderie and M. F. Rivet, Prog. Part. Nucl. Phys. 61 (2008) 551.

[4] E.Bonnet, D. Mercier et al. (INDRA Collaboration), Phys. Rev. Lett. 103 (2009) 072701.

[5] J. Pochodzalla, T. Mhlenkamp et al. (ALADIN Collaboration), Phys. Rev. Lett. 75 (1995) 1040.

[6] Y. G. Ma, A. Siwek et al. (INDRA Collaboration), Phys. Lett. B 390 (1997) 41.

[7] J. B. Natowitz, R. Wada et al., Phys. Rev. C 65 (2002) 034618.

[8] P. Chomaz, V. Duflot and F. Gulminelli, Phys. Rev. Lett. 85 (2000) 3587.

[9] T. Furuta and A. Ono, Phys. Rev. C 74 (2006) 014612.

[10] S. Piantelli, N. Le Neindre et al. (INDRA Collaboration), Phys. Lett. B 627 (2005) 18.

[11] S. Piantelli, B. Borderie et al. (INDRA Collaboration), Nucl. Phys. A 809 (2008) 111.

[12] A. H. Raduta and A. R. Raduta, Nucl. Phys. A 703 (2002) 876.

[13] S. E. Koonin and J. Randrup, Nucl. Phys. A 474 (1987) 173.

[14] Y. D. Kim, R. T. de Souza et al., Phys. Rev. C 45 (1992) 338.

[15] G. Tăbăcaru, M. F. Rivet et al. (INDRA Collaboration), Nucl. Phys. A 764 (2006) 371.

[16] A. S. Goldhaber, Phys. Lett. B 53 (1974) 306.

[17] W. Bauer, Phys. Rev. C 51 (1995) 803.

[18] H. Zheng and A. Bonasera, Phys. Lett. B 696 (2011) 178. 